\title{
EFFECT OF HIGH-FRUCTOSE SOLUTION ON BODY WEIGHT, BODY FAT, BLOOD GLUCOSE AND TRIGLYCERIDE LEVELS IN RATS
}

\author{
Rositsa V. Sandeva, \\ Stanislava M. Mihaylova, \\ Gergana N. Sandeva ${ }^{1}$, \\ Katya Y. Trifonova, \\ Ruska D. Popova-Katsarova ${ }^{2}$ \\ Department of Physiology, \\ Pathophysiology and Pharmacology, \\ Medical Faculty, Trakia University \\ ${ }^{1}$ Department of Hygiene, Infectious \\ Diseases, and Epidemiology, \\ Medical Faculty, Trakia University \\ ${ }^{2}$ Clinical Laboratory, \\ First Internal Clinic, \\ Medical Faculty, Trakia University, \\ Stara Zagora
}

\author{
Corresponding Author: \\ Rositsa V. Sandeva \\ Department of Physiology, \\ Pathophysiology and Pharmacology \\ Medical Faculty, Trakia University \\ 11 Armeyska str. \\ Stara Zagora, 6000 \\ Bulgaria \\ e-mail: rossisandeva@yahoo.com
}

Received: December 10, 2014

Revision received: January 15, 2015

Accepted: May 18, 2015

\begin{abstract}
Summary
In Europe, as well as in Bulgaria, consumption of soft drinks and confectionery has increased during the last three decades and is partly responsible for the epidemiclike increase in obesity. These foods, originally sweetened by sucrose, are now sweetened by other caloric sweeteners such as fructose. In this study we investigated the effect of an eight-week intake of $20 \%$ fructose solution on body weight in rats. Two adult rat groups (aged $120 \pm 6$ days) of Wistar line were studied: a Control group (C; $\mathrm{n}=10 ; 5$ male and 5 female rats) received water and standard rodent chow, and a Fructose group (F; n=12; 6 male and 6 female rats) who received $20 \%$ fructose-indrinking-water solution and regular rodent chow. All animals were weighed and measured (nose to anus length), and the Lee index (equivalent of BMI in rats) was calculated. Body fat was also analyzed. As indicators of increased caloric intake of the Fructose group we investigated glucose, triglycerides and cholesterol (total, HDL and LDL) in blood. In conclusion, consumption of fructose solution in rats resulted in increased body weight, length and measured body fat, increased blood glucose, total cholesterol and triglycerides in the Fructose group, as compared to the controls.
\end{abstract}

Key words: high-fructose diet, obesity, body weight, blood glucose, blood triglycerides

\section{Introduction}

In Europe, as well as in Bulgaria, consumption of soft drinks and confectionery has increased for the last decades and is partly responsible for the epidemic-like increase of metabolic syndrome, type 2 diabetes, obesity and nonalcoholic fatty liver disease (NAFLD) [1-3]. These foods, originally sweetened by sucrose, are now sweetened by other caloric sweeteners such as fructose. While virtually absent in our diet a few hundred years ago, fructose has now become a major constituent of modern diet. The main sources of fructose are sucrose from beet or cane, fruits, honey and high fructose corn syrup (HFCS). In the modern Western society the prevalent sweetener is high fructose corn syrup, known in Bulgaria as glucose-fructose syrup [1,4-7].

Rats are commonly used as an experimental 
model of metabolic syndrome in humans. Studies on rats on a high-fructose diet have shown glucose intolerance, insulin resistance, hyperinsulinaemia and dyslipidaemia [8-10].

Humans and animal models exposed to highfructose diet express different metabolic disorders. In rats, the differences are determined by their breed - Wistar or Sprague-Dawley; the quantity and route of administration of fructose $60 \%$ fructose diet, oral $(8 \mathrm{~g} / \mathrm{kg})$, or mixed in the drinking water $(10 \%, 15 \%$ solution $)$; sex and age of the animals - young or old. The length of these experiments is not standardized - they can last from four weeks to several months [4, 8-13].

Most commonly used and widely described in literature are experiments with $60 \%$ fructose diet (leading to significant metabolic disorders), and with 10\% fructose solution (resulting in less pronounced metabolic disorders). Therefore, we decided to investigate the effect of an 8-week administration of $20 \%$ fructose solution (in drinking water) on glucose and lipid metabolism in 4-month-old Wistar rats of both sexes.

\section{Materials and Methods}

Twenty-two rats (11 male and 11 female) weighing 275-375 g were studied. The control group $\mathrm{C}(\mathrm{n}=10 ; 5$ male and 5 female) received drinking water and standard rodent chow.

The experimental fructose-fed group $\mathrm{F}(\mathrm{n}=12$; 6 male and 6 female) received solution of $20 \%$ fructose in drinking water and the same food ad libitum.

The rats were kept in the accredited vivarium of the Medical Faculty, Trakia University at $25 \pm 1^{\circ} \mathrm{C}$ with a photoperiod light/dark of $12 / 12$ hours and free access to water and food. The containers with water and $20 \%$ fructose solution were supplemented every two days, once a week the amount consumed by both groups was calculated and the average intake of fructose in $\mathrm{g} / 100 \mathrm{~g}$ body weight per day for the experimental group was determined. Weight of all was measured at the start of the experiment and at the end of the 2nd, 4th, 6th and 8th week. At the beginning and end of the experimental period, the length of the rats (distance nose-anus) was measured and the index Lee was calculated. This index in rats is acknowledged as an equivalent to the body mass index in humans. 1000

Lee index $=\sqrt[3]{ }$ body weight $(\mathrm{g}) /$ length $(\mathrm{cm}) \times$
The experiment was conducted in compliance with the requirements of national legislation and the European Directive 2010/63/EU of 22.09.2010 on the protection of animals used for scientific purposes.

Venous blood was collected from the tail vein at the beginning, middle and end of the experimental period. Glucose and lipid parameters (triglycerides, total cholesterol, HDL and LDL cholesterol) in serum were examined using an automatic analyzer Mindray BS300. Blood samples in K3EDTA containers were also taken for subsequent determination of plasma ghrelin and leptin. At the end of the experiment, the rats were euthanized with Nembutal and depots of retroperitoneal, mesenteric (visceral) and subcutaneous adipose tissue from the back were excised and weighed according to Cinti in $\mathrm{mg} / 100 \mathrm{~g}$ body weight [14]. All results were analyzed by Student's t-test on Statsoft Statistica v.8.

\section{Results}

Figure 1 shows the changes in body weight of the control and fructose-fed group of rats for the duration of the study. At the initial weighing the control group had an average weight of $323.5 \pm 35.6 \mathrm{~g}$, and at the final $-384 \pm 22.4 \mathrm{~g}$. For the experimental group these values were $328.7 \pm 30.4 \mathrm{~g}$ and $409.6 \pm 13.2$ respectively. There was a statistically significant difference $(p=0.003)$ between mean body weight of the two groups at the $8^{\text {th }}$ week.

The female fructose group showed greater weight gain compared with males of the same group. It might be that the differences in weights of male and female rats in the fructose group were due to lower physical and metabolic activity of female rats. This probably reflects the higher quantity of fat deposits in adult female rats from both groups. Unfortunately, it was not possible to determine the rats' energy consumption and physical activity during the study and confirm this theory. Increase in length of fructose-fed rats was also greater as compared to controls, which resulted in a slightly decreased Lee index in the fructose-fed group (Table 1).

Statistically significant differences were recorded between the control and fructose-fed groups with respect to serum glucose $(p=0.000005)$, total cholesterol $(p=0.000001)$ and TAG $(\mathrm{p}=0.000017)$. 


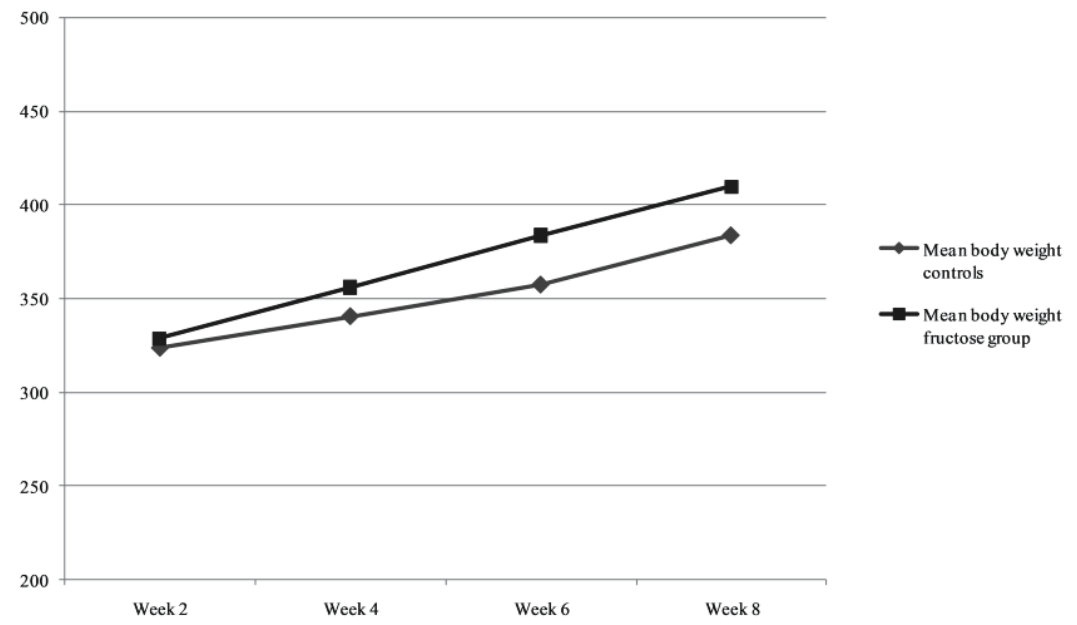

Figure 1. Mean body weight (g) of fructose-fed and control rats

Table 1. Effects of 8 -week administration of $20 \%$ fructose in drinking-water solution in adult male and female Wistar rats

\begin{tabular}{lllll}
\hline Parameter & \multicolumn{2}{c}{ Control group $\mathbf{n = 1 0}$} & \multicolumn{2}{c}{ Fructose group n=12 } \\
& Mean & SD & Mean & SD \\
\hline Serum glucose $(\mathrm{mM})$ & 5.9 & 0.3 & 6.9 & 0.5 \\
\hline Serum total cholesterol $(\mathrm{mM})$ & 2.79 & 1.0 & 6.24 & 0.69 \\
\hline Serum HDL-cholesterol $(\mathrm{mM})$ & 1.2 & 0.17 & 0.89 & 0.09 \\
\hline Serum LDL- cholesterol $(\mathrm{mM})$ & 0.28 & 0.14 & 0.69 & 0.11 \\
\hline Serum TAG (mM) & 1.87 & 0.64 & 3.04 & 0.29 \\
\hline Retroperitoneal fat depot weight $(\mathrm{mg} / 100 \mathrm{~g} \mathrm{bw})$ & 612.1 & 39.4 & 1069.4 & 121.2 \\
\hline Mesenteric fat depot weight $(\mathrm{mg} / 100 \mathrm{~g} \mathrm{bw})$ & 747.8 & 84.1 & 1191.7 & 117.5 \\
\hline Subcutaneous posterior fat depot weight $(\mathrm{mg} / 100 \mathrm{~g} \mathrm{bw})$ & 460.7 & 88.3 & 720.1 & 100.4 \\
\hline Lee index & 310.4 & 4.2 & 309.1 & 2.4 \\
\hline
\end{tabular}

\section{Discussion}

Fructose has the same chemical formula as glucose $\left(\mathrm{C}_{6} \mathrm{H}_{12} \mathrm{O}_{6}\right)$, but its metabolism differs markedly from that of glucose due to its almost complete hepatic extraction and rapid hepatic conversion into glucose, glycogen, lactate, and fat. Hepatic de novo lipogenesis and lipotoxicity, oxidative stress, and hyperuricemia have all been proposed as mechanisms responsible for these adverse metabolic effects of fructose $[1,15,16]$.

Such changes in the lipid profile after 4-week administration of $60 \%$ fructose diet in 3-monthold male Wistar rats were established by De Moura et al., but application of $10 \%$ fructose solution for 8 weeks did not result in statistically significant difference between test and control groups [8].
Increased intake of fructose in our experimental group ( $3.4 \mathrm{~g} / 100 \mathrm{~g}$ body weight daily) resulted in severe metabolic changes and metabolic syndrome. According to the same authors, higher intakes of fructose $(3.75 \mathrm{~g} / 100 \mathrm{~g}$ body weight per day) did not lead to the development of metabolic disorders in young 28 -day-old rats. This may be explained with the influence of protective mechanisms in young animals [8].

In relation to our future study of neuropeptides leptin and ghrelin in blood samples from rats, overconsumption of fructose can also be linked to disorders in the peripheral and central regulation of appetite. High fructose diets administered to rats for over two weeks decrease leptin effect and cause peripheral leptin resistance. According to Tappy et al., the anorexic effect of intraperitoneally administered leptin in rats on a fructose diet is eliminated, 
which corre-sponds to a significant decrease of the hypothalamic signal transducer and activator of transcription-3 (STAT-3) phosphorylation in response to fructose [1].

High-fructose diet causes hepatic leptin resistance by increasing the amounts of the cytokine 3 suppressor and reduction of the serine/threonine phosphorylation of key proteins in leptin action and results in fructose-induced nonalcoholic fatty liver disease (NAFLD).

Lindgvist et al. established a $40 \%$ increase in serum ghrelin after 2-week administration of fructose solution in rats, which is interpreted by the authors as a result of hyperphagia and receiving of signals for continuous consumption under the influence of fructose [4]. This leads to activation of the reward system in the hypothalamus, which is confirmed by the up-regulation of CB1 (endocannabinoid receptors) mRNA expression after 2-week intake of fructose and downregulation of NPY mRNA.

\section{Conclusions}

In summary, rats maintained on a fructose-rich diet for 8 weeks showed abnormal weight gain, increased circulating triglycerides, blood glucose and fat deposits.

Fructose over-consumption increases body weight, changes food consumption pattern and lipid profile. It could be partly responsible for the epidemic increase in obesity and metabolic syndrome in the modern Western world. Highfructose diet impairs secretion and production of neuropeptides such as ghrelin and leptin that are involved in the regulation of appetite, and probably activates the reward system in the hypothalamus.

The presented experiment will be included in a larger investigation on the changes in blood levels of these two neuropeptides under the influence of diets with sucrose substitutes.

\section{References}

1. Tappy L, Le KA. Metabolic Effects of Fructose and the Worldwide Increase in Obesity. Physiol Rev. 2010;9091):23-46.

2. Stanhope KL, Griffen SC, Bair BR, Swarbrick MM, Keim NL, Havel PJ. Twenty-four hour endocrine and metabolic profiles following consumption of high fructose corn syrup-, sucrose- fructose-, and glucose-sweetened beverages with meals. Am J Clin Nutr. 2008;87(5):1194-203.
3. Stanhope KL, Havel PJ. Fructose consumption: potential mechanisms for its effects to increase visceral adiposity and induce dyslipidemia and insulin resistance. Curr Opin Lipidol. 2008;19(1):16-24.

4. Lindqvist A, Baelemans A, Erlanson-Albertsson C. Effects of sucrose, glucose and fructose on peripheral and central appetite signals. Regul Pept. 2008;150(1-3):26-32.

5. Bray GA, Nielsen SJ, Popkin BM. Consumption of high-fructose corn syrup in beverages may play a role in the epidemic of obesity. Am J Clin Nutr. 2004;79(4):537-43.

6. Stanhope KL, Schwarz JM, Keim NL, Griffen SC, Bremer AA, Graham JL. Consuming fructosesweetened, not glucose-sweetened, beverages increases visceral adiposity and lipids and decreases insulin sensitivity in overweight/obese humans. J Clin Invest. 2009;119(5):1322-34.

7. Bray GA. Fructose: should we worry? Int J Obesity. 2008;32:S127-31.

8. De Maura RF, Ribeiro C, de Oliveira JA, Stevanato E, de Mello MA. Metabolic syndrome signs in Wistar rats submitted to different high-fructose ingestion protocols. Br J Nutr. 2009;101(8):117884.

9. Bocarsly ME, Powell ES, Avena NM, Hoebel BG. High-fructose corn syrup causes characteristics of obesity in rats: increased body weight, body fat and triglyceride levels. Pharmacol Biochem Behav. 2010;97(1):101-6.

10. Zarfeshani A, Sokhini M, Mutalib A, Khaza'ai H. Evaluating of high fructose diet to induce hyperglycemia and its inflammatory complications in rats. Pak J Nutr. 2012;11(1):21.

11. Alzamendi A, Giovambattista A, Raschia A, Madrid V, Gaillard RC, Rebolledo O. Fructoserich diet-induced abdominal adipose tissue endocrine dysfunction in normal male rats. Endocrine. 2009;35(2):227-32.

12. Martines C, Gonzales E, Garcia RS, Salas G, Constantino-Casas F, Macias L et al. Effects on body mass of laboratory rats after ingestion of drinking water with sucrose, fructose, aspartame, and sucralose additives. Open Obes J. 2010;2:116-24.

13. Abdullah M, Riediger NN, Chen Q, Zhao Z, Azordegan N. Effects of long-term consumption of a high-fructose diet on conventional cardiovascular risk factors in Sprague-Dawley rats. Mol Cell Biochem. 2009;327(1-2):247-56.

14. Cinti S. The adipose organ. Prostaglandins Leukot Essent Fatty Acids. 2005;73(1):9-1.

15. George A. Bray GA, Popkin BM. Dietary Sugar and Body Weight: Have We Reached a Crisis in the Epidemic of Obesity and Diabetes? Diabetes Care. 2014;37(4):950-6.

16. Sievenpiper JL, de Souza RJ, Mirrahimi A, Yu ME, Carleton AJ, Beyene J, et al. Effect of fructose on body weight in controlled feeding trials: a systematic review and meta-analysis. Ann Intern Med. 2012; 156(4):291-304. 